\title{
Ovarian Pregnancy after IVF and Embryo Transfer - Case Report
}

\author{
Laura Keisa *,**, Zane Grabe *,***, Dace Rezeberga *,***** \\ * - Riga Stradiņš University, Riga, Latvia \\ ** - Riga Maternity Hospital, Riga, Latvia \\ *** - Riga East Clinical University Hospital, Riga, Latvia
}

\section{Summary}

Ovarian pregnancy is one of the rarest types of ectopic pregnancy. The first case of it was reported by St. Maurice in 1689, and since then, few cases have been described in the literature. The incidence of ovarian pregnancy has recently increased due to the wider use of intrauterine devices and assisted reproductive techniques. The authors discuss the case of a 36-year-old primigravid woman who presented with abdominal pain, vomiting and amenorrhea. The patient has a two year history of primary infertility treated by in vitro fertilization. The ovarian pregnancy as the initial diagnosis was made during the operation and the final diagnosis was confirmed by the histopathological finding, which fulfilled Spiegelberg criteria.

Key words: ectopic pregnancy, ovarian pregnancy, laparoscopy

\section{AIM OF THE DEMONSTRATION}

The aim of this article is to demonstrate the clinical challenge to diagnose the ovarian pregnancy.

\section{CASE REPORT}

A 36-year-old nullipara woman with complaints of severe lower abdominal pain, vomiting and a history of amenorrhea for 9 weeks was admitted to the emergency department in January 2015. The symptoms had suddenly started 12 hours before.

The patient had a 2-year history of primary infertility due to endometriosis and had undergone two in vitro fertilization (IVF) cycles in 2014 with a negative outcome. The last cycle was done in December 2014 after the induction of ovulation by GNRH antagonist. After the ovarian puncture, an embryo transfer (ET) was done. Two weeks after ET, the $\beta$ hCG was negative. The examination disclosed pallor, pulse $110 / \mathrm{min}$, BP $85 / 45 \mathrm{mmHg}$, tenderness in the lower part of abdomen and the left shoulder pain. The quantitative beta hCG was $1904 \mathrm{mIU} / \mathrm{mL}$, the hemoglobin level - 9,52 g/dL and the RBC - 3,02 $10^{12} / 1$. The vaginal examination showed a normal uterine size, cervical motion and adnexal tenderness, no palpable mass. No gestational sac was seen inside the uterus during ultrasonography. Both ovaries were enlarged $(7 \times 6 \mathrm{~cm})$ with multiple cysts $(\varnothing 2,2-2,5$ $\mathrm{cm})$. Free fluid was seen in the cul de sac. The provisional diagnosis was ectopic pregnancy, hemoperitoneum, and hemorrhagic shock. The laparoscopy was performed. Intraoperative findings revealed normal tubes, and a massive hemoperitoneum. The left ovary was enlarged $(10 \times 5 \mathrm{~cm})$ with multiple cysts connected to the uteroovarian ligament. After separation of adhesions, a nodular lesion on the left ovarian surface was noted. A wedge resection of the nodular lesion was performed. The left ovary was still bleeding after coagulation and the adnexectomy was performed. The estimated blood loss during operation was $4500 \mathrm{ml}$. The Cell Saver system collected $2700 \mathrm{ml}$ of blood. The patient received 2 units of packet blood transfusion, and 2 units of fresh frozen plazma.

The histopathological examination showed left ovarian pregnancy. The intraoperative and the pathological findings fulfilled the Spiegelberg criteria, formulated in 1878, for the correct diagnosis of ovarian pregnancy. The postoperative period was unremarkable and the patient was discharged 5 days after the surgery.

\section{DISCUSSION}

The ovarian pregnancy is one of the rarest types of ectopic pregnancies. It populates only $0.3-3 \%$ of all ectopic pregnancies (3). The ovarian pregnancy can be classified as a primary, when fertilization takes place in an ovary, and as a secondary, when fertilization takes place in the tube and the conceptus is later regurgitated to be implanted in the ovarium stroma (6). Usually it terminates in rupture during the first trimester. However, some cases in literature have reported an ovarian pregnancy that has progressed to full term delivery (7).

Risk factors for ovarian pregnancy are poorly studied. Some authors believe them to be the same as for the tubular pregnancy, for example, assisted reproductive techniques, intrauterine contraceptive device, pelvic inflammatory disease, endometriosis and smoking. However, some researchers consider that the risk factors for ovarian pregnancy are different from the traditional risk factors for tubular pregnancy. In studies it has been found that in vitro fertilization-embryo transfer (IVFET) and use of current intrauterine device (IUD) are the risk factors of ovarian pregnancy (12). The incidence of ovarian pregnancy following IVF-ET has been much higher than following natural conception, respectively $6 \%$ and $3 \%$ of all ectopic pregnancies (12). The reverse 
migration of transferred embryos towards the fallopian tube could explain the ovarian pregnancy after ET (1). The reverse migration could happen due to junctional zone contraction and strong endometrial waves in the fundal area of the uterus, which can be caused by the difficult ET, the manipulation with the tissue forceps, the high volume and pressure of the culture and the tilt-down position of the patient during ET $(1,5)$. Some authors have reported fertilization of unrecovered oocytes if the coitus occurred near the day of the oocytes retrieval (1). IUD reduces intrauterine implantation, but does not have the same effect against ovarian pregnancy (11). IUD reduces intrauterine implantation by $99.5 \%$, and tubal implantation by $95 \%$, but has a small effect against ovarian pregnancy (11).

Diagnostics of ovarian pregnancy is still difficult. Patients mainly have symptoms as in case of ectopic pregnancy, such as abdominal pain, amenorrhoea and vaginal bleeding. With few exceptions, the initial diagnosis is made on the operating table and the final diagnosis only based on the histopathological finding on the basis of Spiegelberg's criteria. Spiegelberg suggested four criteria to distinguish ovarian pregnancy from a distal tubal pregnancy. They are (1.) the gestational sac is at the position of the ovary; (2.) the ovary must be connected to the uterus through the utero-ovarian ligament; (3.) the fallopian tube, including fimbria, on the affected side must be intact and separate from ovary; (4.) the histologically proved ovarian tissue in the wall of ectopic pregnancy (10). The differential diagnosis made by ultrasound and during the surgery remains a clinical challenge to distinguish an ovarian ectopic pregnancy from a corpus luteum or hemorrhagic cyst, or ruptured chocolate cyst $(8,9)$. In the presented case, the nodular lesion, which resembles the hemorrhagic ovarian cyst, was seen during the operation.

The treatment has changed during the recent years. In the past, the treatment for ovarian pregnancy was the oophorectomy or salpingo-oophorectomy by laparotomy (4). Nowadays the laparoscopy is the golden standard for both the diagnosis and the treatment (2). The gentlest type of operation is laparoscopic enucleation of the gestational product; it also protects surrounding ovarian tissue (3). In the presented case the wedge resection was performed, but due to severe bleeding, the oophorectomy was done.

In conclusion, ovarian pregnancy is a rare type of ectopic pregnancy which is difficult to detect. Since the clinical presentation is very similar to tubal pregnancy or a complicated ovarian cyst, the preoperative diagnosis is a challenge, and in most of the cases, the final diagnosis is made during the surgery and based on the histopathological findings.

\section{Conflict of interest: None}

\section{REFERENCES}

1. Abusheikha N, Salha O, Brinsden P. Extra-uterine pregnancy following assisted conception treatment // Human Repro Update, 2000; 6(1):80-92

2. Alkatout I. Practical manual for laparoscopic \& hysteroscopic gynecological surgery. Jaaype Brother Medical Publishers; 2013. Ectopic pregnancy; 169189.

3. Aydin T, Yucel B, Aksoy H, Ekemen S. Successful laparoscopic management of a rare complication after embryo transfer: ovarian pregnancy. A case report and up-to-date literature review // Wideochir Inne Tech Maloinwazyjne, 2015; 10(4):574 - 579

4. Choi HJ, Im KS, Jung HJ, Lim KT, Mok JE, Kwon YS. Clinical Analysis of ovarian pregnancy: a report of 49 cases // Eur J Obstet Gynecol Reprod Biol, 2015; 158(1):87-89

5. Dursun P, Gultekin M, Zeyneloglu HB. Ovarian ectopic pregnancy after ICSI-ET: a case report and literature review // Arch Gynecol Obstet, 2008; 278:191 - 193

6. Gon S, Majumdar B, Ghosal T, Sengupta M. Two Cases of Primary Ectopic Ovarian Pregnancy // Online J Health Allied Scs, 2011; 10(1):26

7. Huang J, Jing X, Fan S, Fufan Z, Yiling D, Pixiang $\mathrm{P}$, Xiaomeng $X$. Primary unruptured full term term ovarin pregnancy with live female infant: case report // Arch Gynecol Obstet, 2011; 283(1):31-33

8. Melcer Y, Maymon R, Vaknin Z, Pansky M, Mendlovic S, Barel O, Smorgick N. Primary ovarian ectopic pregnancy: still a medical challenge // J Reprod Med, 2016; 61(1-2):58 - 62

9. Roy J, Babu AS. Ovarian pregnancy: Two case reports // AMJ, 2013; 6(8):406-410

10. Spiegelberg O. Zur casuistic der Ovarialschwangerschaft // Arch Gynaekol, 1878; $13: 73-76$

11. Tehrani HG, Hamoush ZH, Ghasemi M, Hashemi L. Ovarian ectopic pregnancy: A rare case // Iran J Reprod Med, 2014; 12(4):281-284

12. Zhu Q, Li C, Zhao WH, Yuan JJ, Yan MX, Qin GJ, Zhang J. Risk factors and clinical features of ovarian pregnancy: a case-control study // BMJ Open, 2014; 4:e006447

\section{Address:}

Laura Keisa

Riga Maternity hospital

Miera street 45, LV-1013

Riga, Latvia

E-mail: laura.keissa@gmail.com 